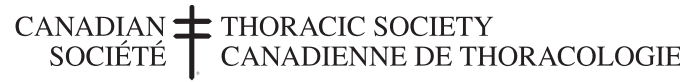

\section{CANADIAN RESPIRATORY CONFERENCE}

The Canadian Thoracic Society (CTS), the Canadian COPD Alliance, the Canadian Respiratory Health Professionals and the Canadian Lung Association are proud to present the Canadian Respiratory Conference (CRC). Join us at the Hilton Montréal Bonaventure Hotel in Montreal, Quebec, June 19 to 21 !

For further details on the CRC, and to register, go to $<$ www.lung.ca/crc $>$.

\section{MEMBERSHIP}

Have you joined the CTS or renewed your membership for the 2008 to 2009 year?

Note the many advantages of membership in the CTS:

- low annual membership fee

- reduced registration fees for the annual American College of Chest Physicians Chest Conference and the annual CRC

- membership fee reduction of $15 \%$ for the European Respiratory Society

- collaboration with a national network of respiratory physicians and researchers promoting lung health across Canada

- CTS social events at the American College of Chest Physicians and American Thoracic Society conferences

- on-line access to the Canadian Respiratory Journal

- educational and practice resources (patient and practice tools, Web sites, guidelines, chronic obstructive pulmonary disease slides)

- participation and leadership opportunities in CTS committees, guideline development and research activities

To join or renew your membership, please visit $<$ www.lung.ca/cts $>$.

\author{
MOVING? PLEASE LET US KNOW - CONTACT: \\ Canadian Thoracic Society \\ 1750 Courtwood Crescent, Suite 300 \\ Ottawa, Ontario \\ K2C 2B5 \\ Telephone: 613-569-6411 ext 270 \\ Fax: 613-569-8860 \\ E-mail: ctsinfo@lung.ca
}

\section{CONGRÈS CANADIEN SUR LA SANTÉ RESPIRATOIRE}

La Société canadienne de thoracologie, L'Alliance canadienne sur la MPOC, Les Professionnels canadiens en santé respiratoire et L'Association pulmonaire sont fiers de présenter le congrès canadien sur la santé respiratoire. Accompagneznous à l'hôtel Hilton Montréal Bonaventure de Montréal, au Québec, du 19 au 21 juin !

Pour obtenir plus d'information au sujet de ce congrès, consultez le site <www.poumon.ca/crc $>$.

\section{ADHÉSION}

Êtes-vous devenu membre de la SCT ou avez-vous renouvelé votre cotisation pour l'exercice 2008-2009?

L'adhésion à la SCT comporte de nombreux avantages :

- une cotisation annuelle peu coûteuse;

- un rabais à l'inscription au congrès annuel de l'American College of Chest Physicians et au congrès canadien annuel sur la santé respiratoire;

- une réduction de $15 \%$ à la cotisation à l'European Respiratory Society;

- la collaboration avec un réseau national de médecins et de chercheurs en santé respiratoire qui font la promotion de la santé pulmonaire au Canada;

- la participation aux activités sociales de la SCT pendant les congrès de l'American College of Chest Physicians et de l'American Thoracic Society;

- l'accès électronique à la Revue canadienne de pneumologie;

- des ressources de formation et des ressources pratiques (outils pour les patients et outils de pratique, sites Web, lignes directrices, présentations sur la maladie pulmonaire obstructive chronique);

- la participation et les possibilités de leadership aux comités de la SCT, à l'élaboration de lignes directrices et aux activités de recherche.

Pour devenir membre ou renouveler votre cotisation, consultez le site < www.poumon.ca/sct>.

\section{VOUS DÉMÉNAGEZ ? FAITES-LE-NOUS SAVOIR :}

Société canadienne de thoracologie

1750, Courtwood Crescent, bureau 300

Ottawa (Ontario)

K2C 2B5

Téléphone : 613-569-6411, poste 270

Télécopieur : 613-569-8860

Courriel : infosct@poumon.ca 


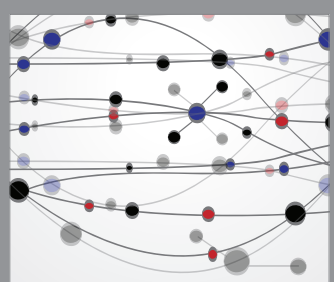

The Scientific World Journal
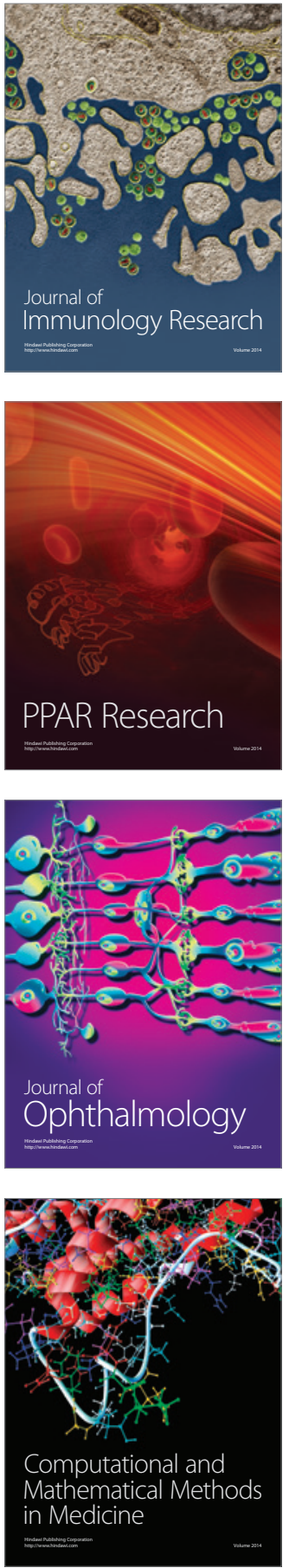

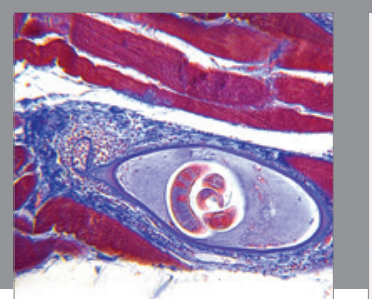

Gastroenterology Research and Practice

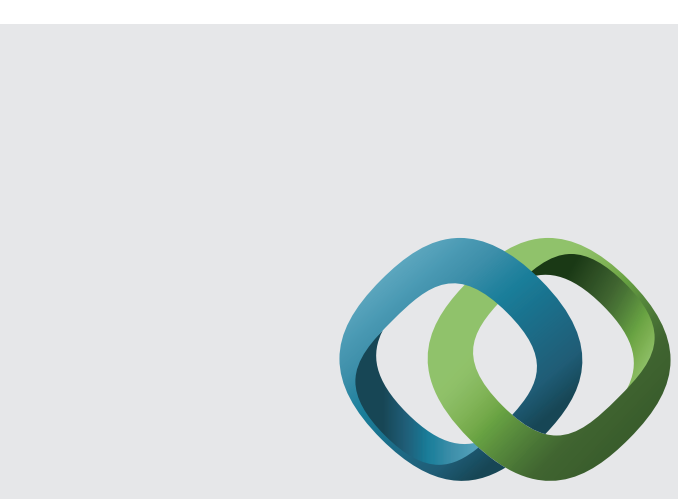

\section{Hindawi}

Submit your manuscripts at

http://www.hindawi.com
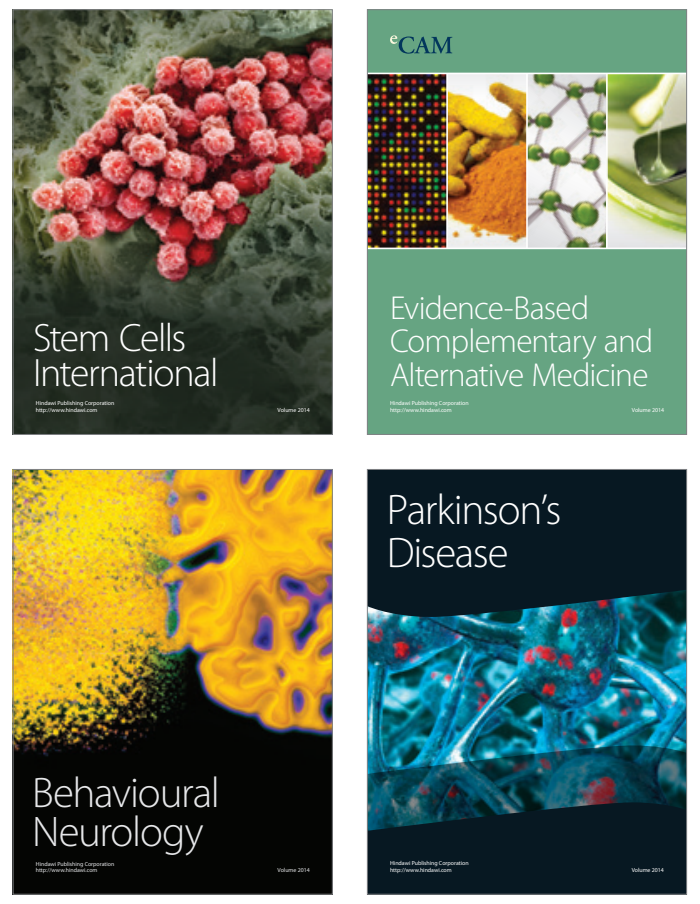
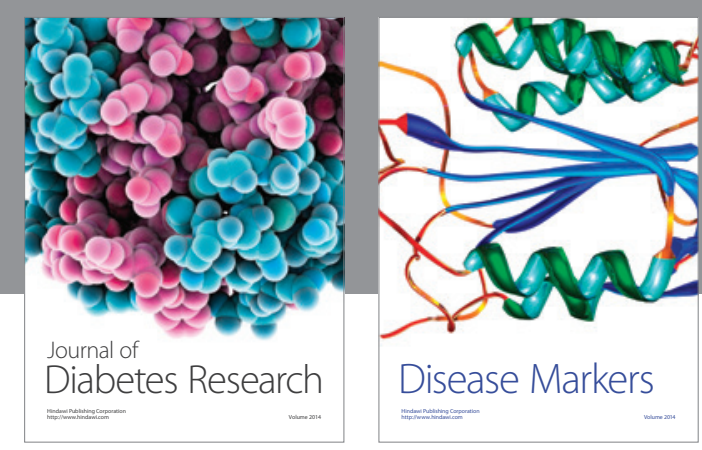

Disease Markers
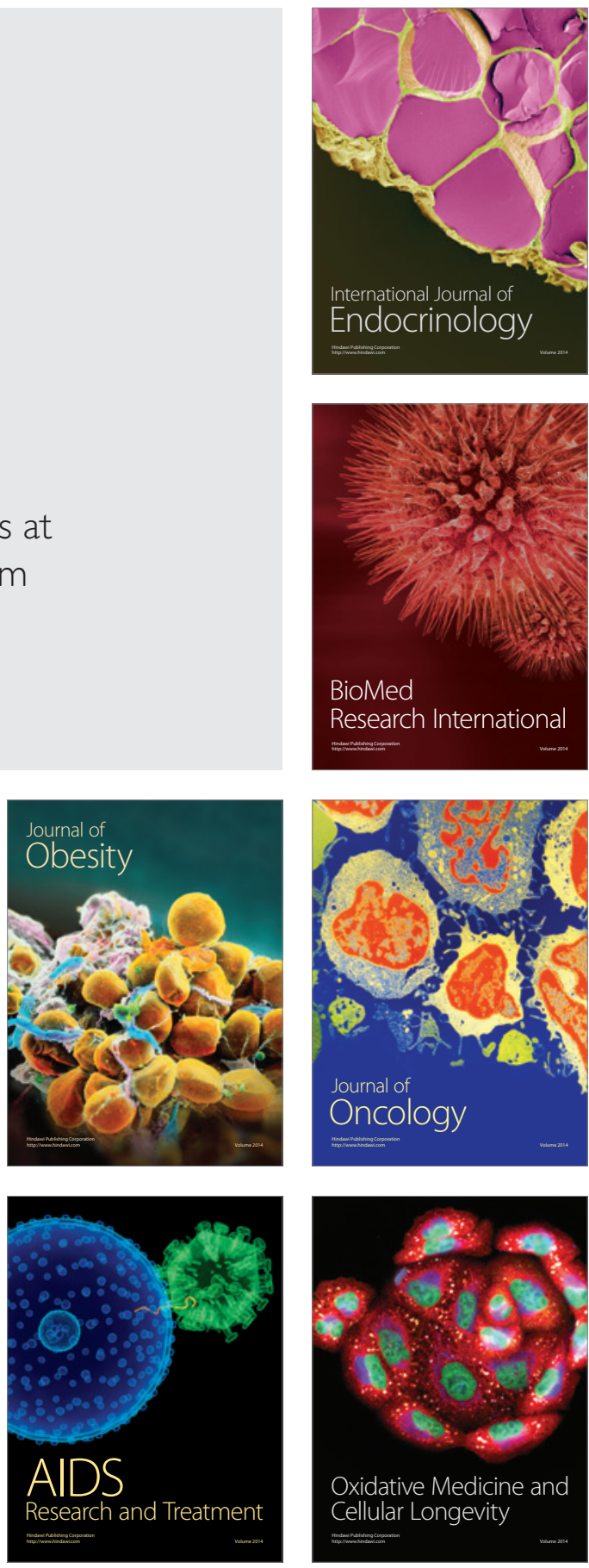